\title{
MOLECULAR MECHANISMS OF THE SYNERGY BETWEEN CYSTEINYL-LEUKOTRIENES AND RECEPTOR TYROSINE KINASE GROWTH FACTORS ON HUMAN BRONCHIAL FIBROBLAST PROLIFERATION
}

\author{
Hajime Yoshisue, Stephen T. Holgate, Donna E. Davies and Anthony P. Sampson \\ Division of Infection, Inflammation and Repair, Southampton University School of Medicine, Southampton, UK
}

WINNING ABSTRACT: We have reported that cysteinyl-leukotrienes (cys-LTs) synergise not only with epidermal growth factor (EGF) but also with platelet-derived growth factor (PDGF) and fibroblast growth factor (FGF) to induce mitogenesis in human bronchial fibroblasts. We now describe the molecular mechanisms underlying this synergism. Mitogenesis was assessed by incorporation of $\left[{ }^{3} \mathrm{H}\right]$ thymidine into DNA and changes in protein phosphorylation by Western blotting. Surprisingly, no CysLT receptor antagonists (MK-571, montelukast, BAY u9773) prevented the synergistic mitogenesis. LTD $_{4}$ did not cause phosphorylation of EGFR nor did it augment EGF-induced phosphorylation of EGFR, and the synergy between LTD $_{4}$ and EGF was not blocked by the metalloproteinase inhibitor GM6001 or by an HB-EGF neutralising antibody. The EGFR-selective kinase inhibitor, AG1478, suppressed the synergy by LTD $_{4}$ and EGF, but had no effect on the synergy with PDGF and FGF. While inhibitors of mitogen-activated protein kinase, phosphatidylinositol 3-kinase and protein kinase $C$ (PKC) prevented the synergy, these drugs also inhibited mitogenesis elicited by EGF alone. In contrast, pertussis toxin (PTX) efficiently inhibited the potentiating effect of $\mathrm{LTD}_{4}$ on EGF-induced mitogenesis, as well as that provoked by PDGF or FGF, but had no effect on mitogenesis elicited by the growth factors alone. Whereas $\mathrm{LTD}_{4}$ alone did not augment phosphorylation of extracellular signalregulated kinase (Erk)-1/2 and Akt, it increased phosphorylation of PKC in a Goi-dependent manner. Addition of LTD 4 prolonged the duration of EGF-induced phosphorylation of Erk-1/2 and Akt, both of which were sensitive to PTX. The effect of cys-LTs involves a PTX-sensitive and PKC-mediated intracellular pathway leading to sustained growth factor-dependent phosphorylation of Erk-1/2 and Akt.

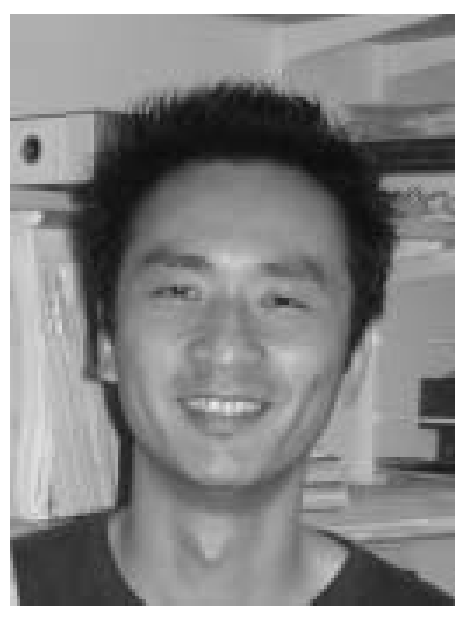

Hajime Yoshisue

Novartis Pharma KK, Tsukuba Research Institute, Tsukuba, Ibaraki, Japan

\section{MY JOB AND THE UNIT IN WHICH I WORK}

I am a postdoctoral research scientist working in the Division of Infection, Inflammation and Repair (IIR), University of Southampton School of Medicine, Southampton General Hospital, Southampon, UK. From November 2003, I have been funded by Asthma UK to work on the molecular mechanisms underlying the effect of cysteinyl-leukotrienes (cys-LTs), lipid mediators closely involved in the pathogenesis of asthma [1], on primary human bronchial fibroblasts established from healthy volunteers. I am currently supervised by Prof. Stephen Holgate, Prof. Donna Davies and Dr Anthony Sampson. In addition to this major work, I have been technically supporting other members of the laboratory for molecular biology works (e.g. cloning), as I have much experience and knowledge, especially in technologies of molecular biology.

\section{MY WINNING POSTER AS PART OF MY RESEARCH}

After gaining a PhD in 1995, I started work as a research scientist in the Tokyo Research Laboratories of Kyowa Hakko Kogyo Co., Ltd, Tokyo, Japan. During my 8 yrs with Kyowa, I worked on the isolation and validation of novel drug targets mainly using the technique of differential cloning. These studies included the following: 1) identification and functional characterisation of a novel human chemokine, eotaxin-3; 2) large-scale isolation of non-uniform shear stress-responsive genes in human endothelial cells by making a subtracted cDNA library; 3) isolation of candidate genes involved in the regeneration of pancreatic $\beta$-cells for stem cell therapy of diabetes; and 4) evaluation of matrix metalloproteinase inhibitors on airway mucous production. Two international patents and three first-author papers were published from these studies.

In 2003, I left Kyowa and moved to the University of Southampton School of Medicine as a Research Fellow, to focus on the molecular mechanisms of the pathogenesis of respiratory diseases, including asthma. My first project in Southampton was the identification and characterisation of a novel human gene, which was less abundant in cDNA libraries 
from asthmatic bronchi than from normal bronchi. This gene, ciliated bronchial epithelium (CBE)1, is strongly induced during ciliogenesis and is highly associated with ciliated cells. Forced overexpression of CBE1 leads to induction of apoptosis, raising the possibility that it causes cell cycle arrest during commitment to differentiation, suggesting that CBE1 may play a key role in ciliogenesis. This work was published in the American Journal of Respiratory Cell and Molecular Biology in 2004 [2]. I am currently focusing my work on signal transduction by cys-LTs in human bronchial fibroblasts (the present winning abstract). This work was chosen as an oral presentation in the Keystone Symposium (USA, January 2006), and the manuscript has been accepted recently for publication in the Journal of Allergy and Clinical Immunology [3].

Although I have been working on different types of disease and in different places, including academia and industry, I have been consistently involved in the analyses of the molecular mechanisms of diseases in order to find novel drug targets and/or novel therapeutic approaches. The present cysLT work is also one of my basic research projects.

\section{MY RESEARCH AS PART OF MY WORKING GROUP/ RESEARCH TEAM}

The Allergy and Inflammation Research (AIR) subdivision of the IIR led by Prof. Stephen Holgate, has been a world-leading asthma research group over the past two decades. This has been achieved through integration of basic and clinical science using well-phenotyped patients across the spectrum of asthma severity and establishment of key collaborations to facilitate translation of new conception to the disease. I work in the Brooke Laboratory where Prof. Donna Davies, Deputy Director of AIR, leads research into mechanisms of asthma and chronic obstructive pulmonary disease. She has a long-standing interest in the epidermal growth factor (EGF) receptor and her current interests are focused on the structural cells of lung (epithelial and mesenchymal cells), which are increasingly recognised to play an important role in airway remodelling. One recent notable achievement is the discovery of a defect in the innate immune response of asthmatic epithelial cells to rhinovirus infection [4, 5]. Other people in the Brooke Laboratory (Southampton, are working on a disintegrin and metalloproteinase 33 (ADAM33), a novel asthma-susceptibility gene [6], which might have a critical role in airway remodelling and/or bronchial hyperresponsiveness. Dr Anthony Sampson is a Reader in Immunopharmacology and has been investigating the interrelationship between respiratory diseases and leukotriene pathways, including 5-lipoxygenase enzymes and cys-LT receptors [7].

The initial hypothesis of the present study (made by my three supervisors) was that cys-LTs, as the predominant G-proteincoupled receptor (GPCR)-active mediators of myocyte contraction in the asthmatic airway, are critical activators of ADAM sheddases, liberating a membrane-bound EGF receptor ligand, leading to proliferation of bronchial fibroblasts/smooth muscle cells. I have found for the first time that while cys-LTs alone do not affect proliferation of human bronchial fibroblasts, in the presence of EGF they markedly augment proliferation, although ADAM proteins are not involved in this process. Since proliferation of airway fibroblasts is an important component of airway

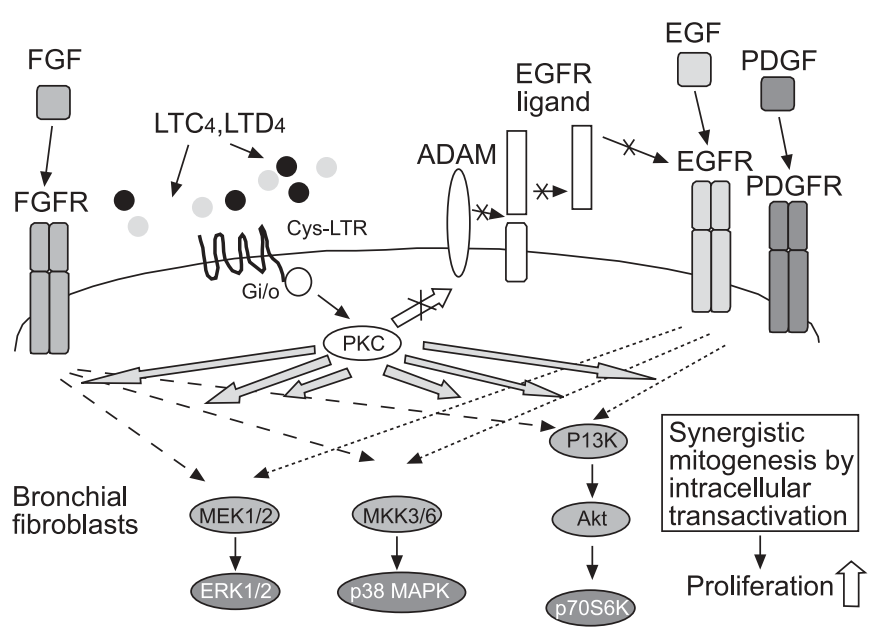

FIGURE 1. Summary of this study. Cysteinyl-leukotrienes (cys-LTs) stimulate mitogenesis of bronchial fibroblasts only in the presence of RTK growth factors, not through transactivation of epidermal growth factor receptor (EGFR) by a disintegrin and metalloproteinase (ADAM)-mediated ligand shedding (indicated by arrows covered by a cross), but through intracellular, protein kinase $C$ (PKC)-mediated augmentation of growth factor-dependent phosphorylation of signalling molecules. FGF(R): fibroblast growth factor (receptor); LT: leukotriene; PDGF(R): plateletderived growth factor (receptor); MEK: mitogen-activated protein kinase (MAPK)/ extracellular signal-regulated kinase (ERK) kinase; MKK: mitogen-activated protein kinase kinase; PI3K: phosphoinositide-3 kinase; Akt: protein kinose B (PKB).

remodelling and is observed in chronic asthma, my research fits well into the major concept of our working group.

\section{THE IMPACT OF MY WORK ON CLINICAL OR RESEARCH PRACTICE}

In the present study, we have shown that cys-LTs synergise with receptor tyrosine kinase growth factors to promote human bronchial fibroblast proliferation via a Gi/o-dependent and protein kinase C (PKC)-mediated intracellular pathway, leading to augmented growth factor-dependent activation of extracellular signal-regulated kinase (Erk)-1/2 and Akt (fig. 1). The effect is unlikely to be mediated by cys-LT1R or cys-LT2R, but may be mediated by another GPCR. These observations may have clinical implications for the long-term actions of cys-LTs in airway remodelling in asthma. Despite the efficient inhibition of airway fibrosis by montelukast in an ovalbumin-challenged mouse model [8], the inability of cys-LT1R-selective antagonists to prevent the synergistic effect by cys-LTs and growth factors on fibroblast proliferation suggests that these therapies may be ineffective in suppressing the fibrotic changes observed in the airways of patients with chronic asthma.

\section{REFERENCES}

1 Holgate ST, Peters-Golden M, Panettieri RA, Henderson WR Jr. Role of cysteinyl leukotrienes in airway inflammation, smooth muscle function, and remodeling. J Allergy Clin Immunol 2003; 111: S18-S36.

2 Yoshisue H, Puddicombe SM, Wilson SJ, et al. Characterization of ciliated bronchial epithelium 1, a ciliated cell-associated gene that is induced during mucociliary differentiation. Am J Respir Cell Moll Biol 2004; 31: 491-500. 
3 Yoshisue H, Kirkham-Brown J, Healy E, Holgate ST, Sampson AP, Davies DE. Cysteinyl-leukotrienes synergize with growth factors to induce proliferation of human bronchial fibroblasts. J Allergy Clin Immunol 2007; 119: 132-140.

4 Wark P, Johnston SL, Bucchieri F, et al. Asthmatic bronchial epithelial cells have a deficient innate immune response to infection with rhinovirus. J Exp Med 2005; 201: 937-947.

5 Contoli M, Message SD, Laza-Stanca V, et al. Role of deficient type III interferon- $\lambda$ production in asthma exacerbations. Nat Med 2006; 12: 1023-1026.
6 Van Eerdewegh P, Little RD, Dupuis J, et al. Association of the ADAM33 gene with asthma and bronchial hyperresponsiveness. Nature 2002; 418: 426-430.

7 Peters-Golden M, Sampson AP. Cysteinyl leukotriene interactions with other mediators and with glucocorticosteroids during airway inflammation. J Allergy Clin Immunol 2003; 111: S37-S42.

8 Henderson WR Jr, Tang LO, Chu SJ, et al. A role of for cysteinyl leukotrienes in airway remodeling in a mouse asthma model. Am J Respir Crit Care Med 2002; 165: 108-116. 